TM-1533

\title{
Prospects for 6 to 10 Tesla Magnets for a TEVATRON Upgrade*
}

\author{
Paul M. Mantsch \\ Fermi National Accelerator Laboratory \\ P.O. Box 500, Batavia, Illinois 60510
}

July 8,1988

*Prepared for the 1988 DPF Summer Study at Snowmass, Snowmass, Colorado, July 8, 1988. 


\title{
Prospects for 6 to 10 Tesla Magnets \\ for a Tevatron Upgrade
}

\author{
Prepared for the 1988 DPF \\ Summer Study at Snowmass
}

Paul M. Mantsch

Fermilab

The first SSC physics is at least 10 years away. An upgrade of the Fermilab Tevatron will ensure the continuity of a vigorous highenergy physics program until the SSC turns on. Three basic proposals are under consideration:

1) $\bar{p}$ at $3 \times 10^{31}$ - Increase luminosity by improvements to the

2) $\mathrm{pp}$ at $1 \mathrm{TeV}$ and $2 \times 10^{32}$ - Move the main ring to a new tunnel, build a second Tevatron ring. 3) $\overline{\mathrm{p} p}>1.5 \mathrm{TeV}$ and $7 \times 10^{30}-$ Replace the Tevatron with a

The last two options require new higher-field magnets. The second option requires about a hundred 6.6-tesla dipoles in addition to a ring of Tevatron strength $(4.4 \mathrm{~T})$ magnets. These higher-field magnets are necessary in both rings to lengthen the straight sections in order to realize the collision optics. The third option requires a ring of magnets of $6.6 \mathrm{~T}$ or slightly higher to replace the present Tevatron plus a number of special 8 - 9 tesla magnets.

The viability of the high-energy option then depends on the practicality of sizable numbers of reliable $8-9$ tesla dipoles as well as 800 6.6-tesla dipoles. The following discussion develops a specification 
for an $8.8 \mathrm{~T}$ dipole, examines the design considerations and reviews the current state of high-field magnet development.

\section{Specifications for 6.6 and 8.8 Tesla Dipoles}

Coil designs under consideration here are of the traditional Tevatron style with two shells wound of Rutherford cable around a circular aperture. The coils are supported by laminated collars. The design differs from the Tevatron in that a cold-iron yoke is used. In fact the cold iron becomes a vital support for the collared coil in highfield magnets.

A conceptual design has been made for a 6.6 tesla magnet as part of the study for a pp collider. The aperture is the same as that of the Tevatron (3.0 inches) and the conductor is that developed for the $\mathrm{D} \emptyset$ low-beta quadrupole system. The specification for this magnet is shown in Table I, a cross section in Fig. 1 and the operating load line in Fig. 2. The cryostat is of the style developed at Fermilab for the SSC. The 6.6 $\mathrm{T}$ magnet, if conservatively designed, can successfully be built. Although designed for 5 tesla the similar HERA magnets operate easily up to 6.9 tesla. This is a proof of principle that such magnets can be built.

The design of an 8.8 tesla magnet, on the other hand, plunges into largely uncharted territory. The high-current densities required assume $1.8 \mathrm{~K}$ operation. At $8.8 \mathrm{~T}$ the forces which must be accommodated are a factor of four higher than in the Tevatron coil. The two most important magnet design considerations are: to achieve high-current density, and to ensure properly loaded coils for stability. The requirement of low temperature, of course, assumes that a practical large-scale refrigeration system for magnet operation in superfluid helium can be built.

Table II shows a proposed specification for an 8.8 tesla magnet. Since this machine is to be used to supply beams for fixed-target operation, the design must be capable of ramping. The aperture is the same as that of the Tevatron ( 3 inches) to permit extraction.

\section{Design Considerations for an 8.8 T Dipole}

The development of the SSC dipole over the past four years and more recently the high-gradient quadrupoles for the low-beta program at Fermilab have lead to important improvements in magnet design and fabrication. These improvements provide a basis for advancing to higher fields. Features critical to a successful design will be considered.

\section{Conductor}

High field is achieved by high-current density. Motivated largely by the needs of the SSC, the advance in current density in $\mathrm{NbTi}$ conductor has been dramatic in the last few years. Figure 3 shows the 


\title{
Proposed Specification for a
}

\author{
6.6 Tesla Dipole for the
}

\author{
Tevatron Upgrade
}

Aperture

Operating field

Operating current

Maximum field

Maximum ramp rate

Operating temperature

Amp factor

Sextupole

Inner coil radius

Outer coil radius

Yoke inner radius

Yoke outer dimensions
3 in.

6.6 tesla

4366 amp

7 tesla

$0.2 \mathrm{~T} / \mathrm{sec}$

$4.6 \mathrm{~K}$

$100.855 \%$

$4.4 \times 10^{-4}$

$1.50 \mathrm{in}$.

2.29 in.

3.54 in.

$12.6 \times 18.1 \mathrm{sq}$ in. 


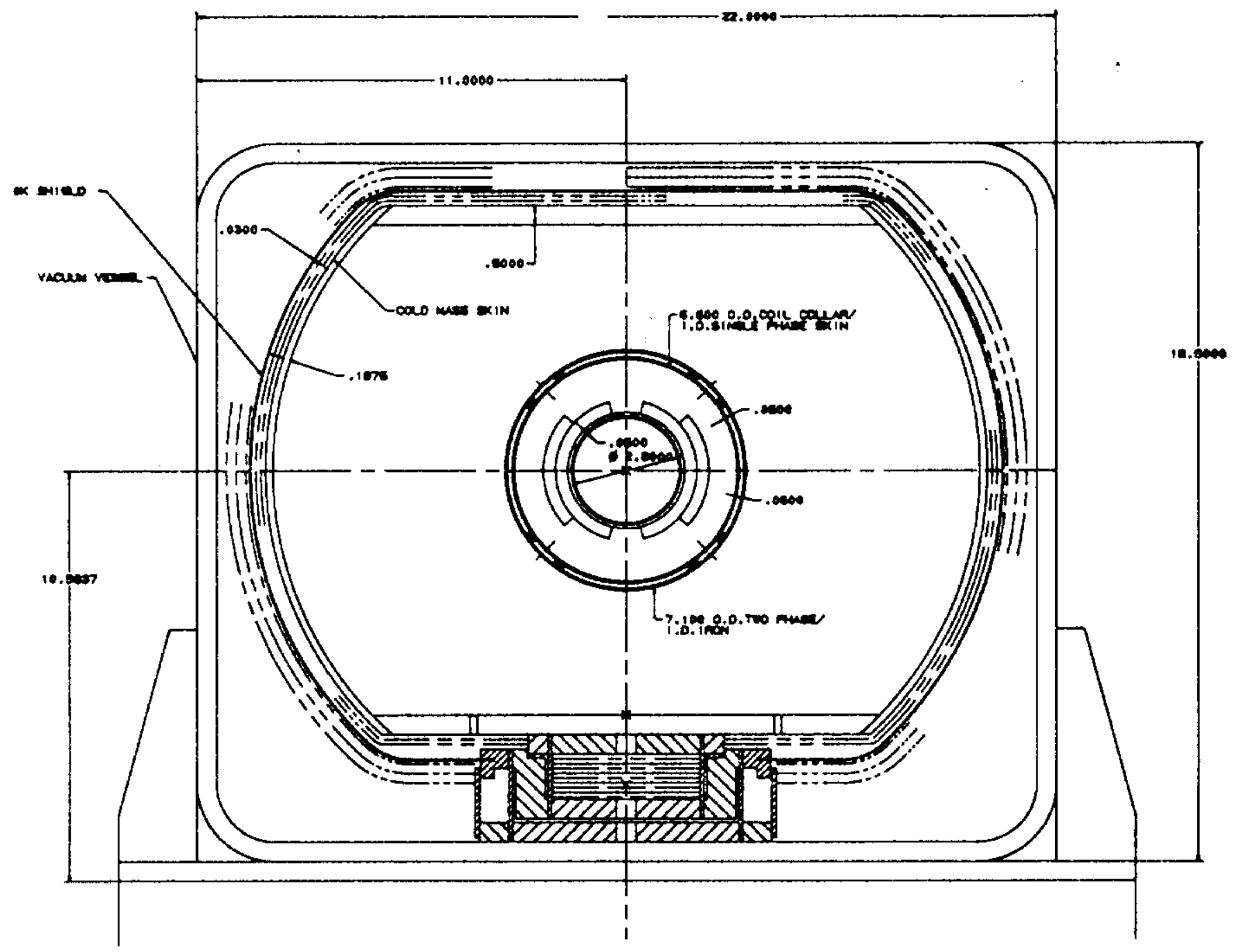

Figure 1

6.6 Tesla Dipole Cross Section 


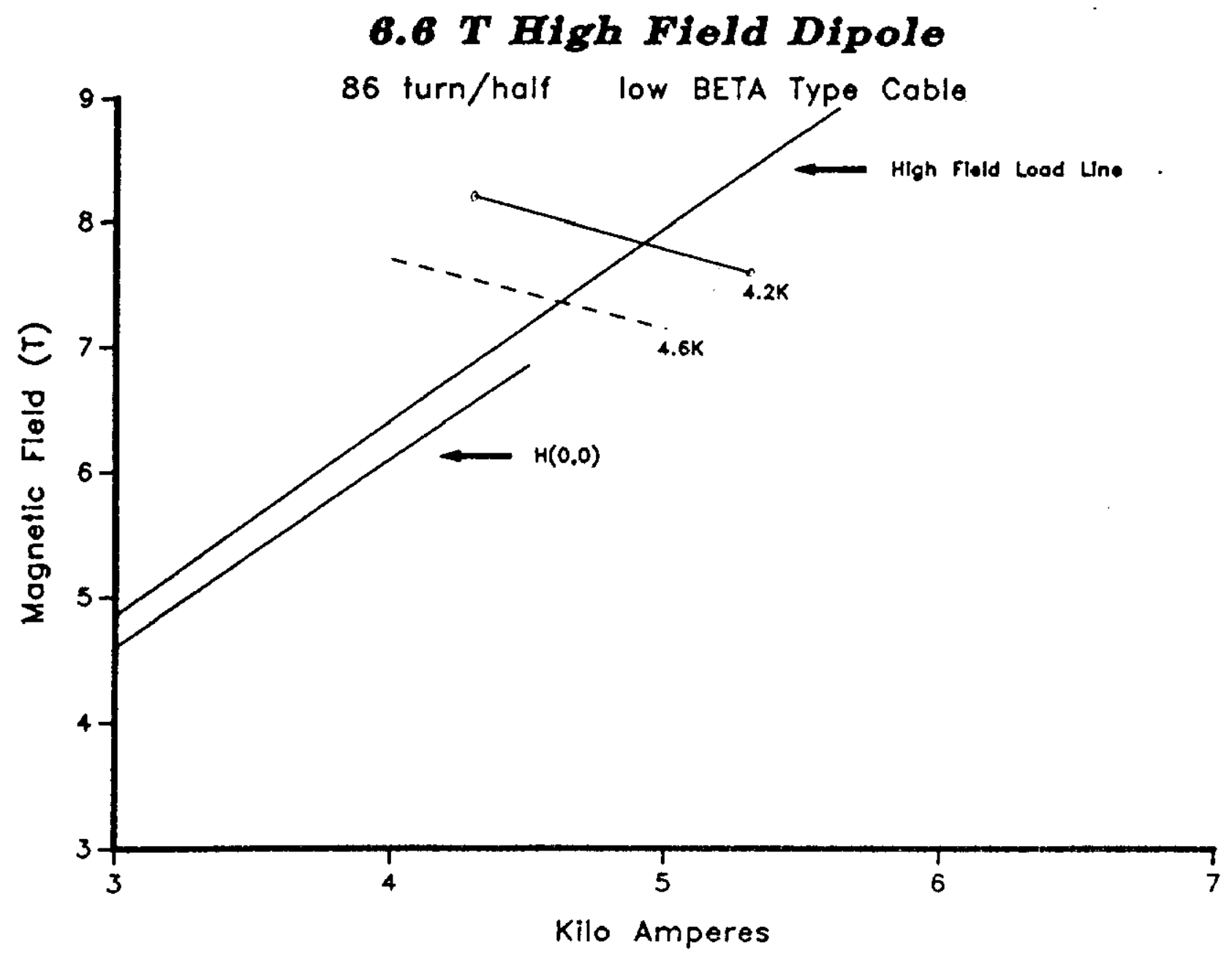

Figure 2

6.6 Tesla Dipole Load Line 


\title{
Proposed Specification for an
}

\author{
8.8 T Dipole for the
}

Tevatron Upgrade
Aperture
3 inches $(7.62 \mathrm{~cm})$
Operating field
8.8 tesla
Maximum field
10.0 tesla
Maximum ramp rate
$0.2 \mathrm{~T} / \mathrm{sec}$
Operating temperature
$1.8 \mathrm{~K}$
Saturation sextupole
$<5$ units@ $9.8 \mathrm{~T}$
Amp factor
$<1.05 @ 8.8 \mathrm{~T}$

Table II 


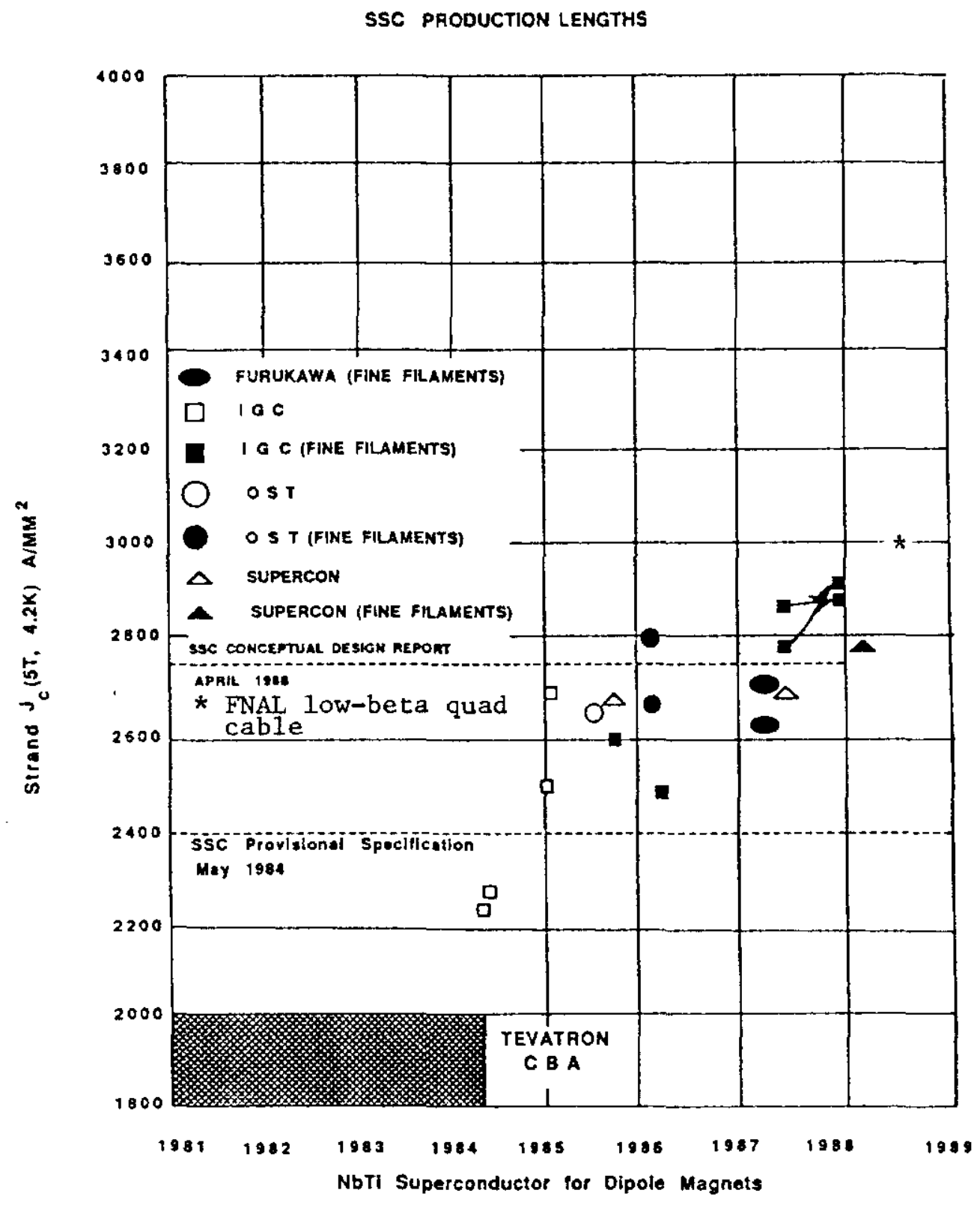

Figure 3

Current Density Improvements 
increase in current density in the superconductor achieved over the past few years. ${ }^{2}$ The current density specification for the Tevatron was $1800 \mathrm{~A} / \mathrm{mm}^{2}$ at $5 \mathrm{~T}, 4.2 \mathrm{~K}$ in cable form. The most recent cable received for the low-beta quad program has a current density in the superconductor of $2914 \mathrm{~A} / \mathrm{mm}^{2}$ - a $60 \%$ improvement! This increase has resulted from intensive study of the microscopic structure of superconductor by David Larbalestier and co-workers at the University of Wisconsin. A highly homogenous alloy melt, together with a carefully developed schedule of cold work and heat treatments, is responsible for these improvements.

At low temperatures and high fields, NbTiTa alloy conductor has offered a current density improvement over NbTi. Although more development needs to be done, existing NbTiTa alloy could add at least a half tesla to the field of a magnet at 9 tesla and $2 \mathrm{~K}$.

The characteristic (critical current vs. applied field) for the lowbeta quadrupole conductor is shown in Fig. 2. The performance of a magnet is determined by drawing a "load line" from the origin through a point representing the field at the high-field point on the coil and the current at that field. The point where the load line intersects the critical current characteristic of the conductor is the short sample limit of the magnet. The aperture field is 6 to $9 \%$ lower than the high-field point line at the same current. It is clear that the field goes up more slowly than the critical current density as the field must follow the load line.

A secondary goal is high-current density in the cable as a whole. Current density in the cable therefore depends on the copper to superconductor ratio. There is not a clear understanding of the role of the copper content (or the properties of the copper) in the stability of a finished magnet, however. Stability is measured (rather poorly) by the number of quenches necessary to reach the conductor short sample limit. Over 30 models of SSC magnets have been built with copper to superconductor ratios varying from 1.28:1 to 1.6:1. The test data do not do much to illuminate this issue. We have excellent performers with 1.30:1 and with 1.6:1. Differences in performance as measured by quench history are apparently obscured by other effects.

Sampson has measured large numbers of cable samples at Brookhaven. The sample is supported rigidly in a fixture that is inserted into the bore of a high-field dipole. A measurement is then made of the characteristic of the cable. Many of the cables quench several times before reaching a plateau current. Sampson finds the number of cable quenches to be a strong function of the copper to superconductor ratio. A sketch of the cable support fixture and a plot of quench number vs. current for a number of SSC cables is shown in Fig. $4 .^{3}$ In fact, when magnets are made of these cables the quench performance is not nearly as bad as predicted by Sampson's plot. For example a cable with a ratio of $1.3: 1$ requires about 10 quenches to reach a plateau in Sampson's measurement. A 1 meter model wound with this cable, D-15-A3, reaches that short sample limit on the second quench. The quench history of D-15A-3 is shown in Fig. 5. 

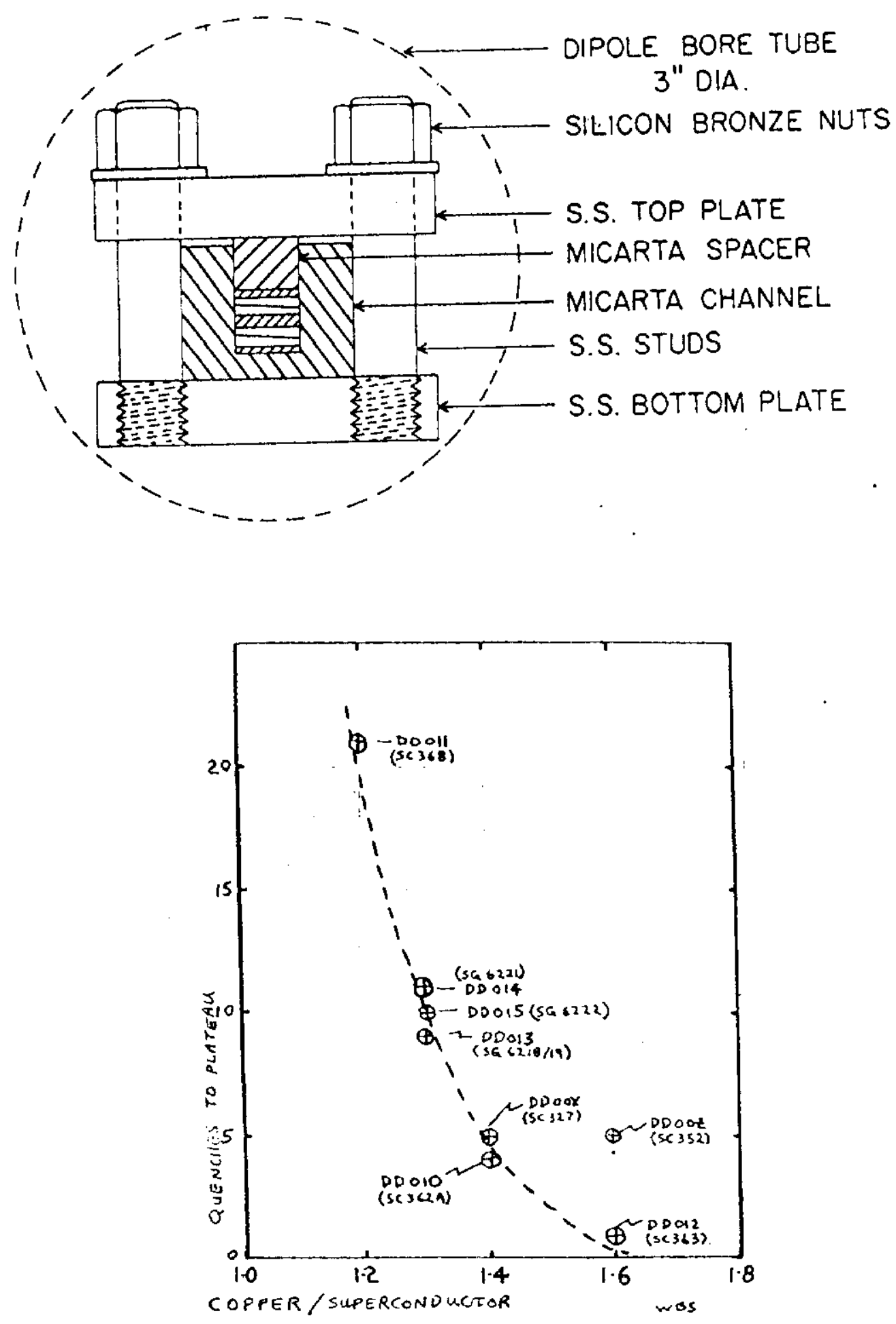

Figure 4

a) BNL fixture for a cable short sample measurement

b) Short sample measurements of the SSC cables. Quench sensitivity to copper to superconductor ratio. 


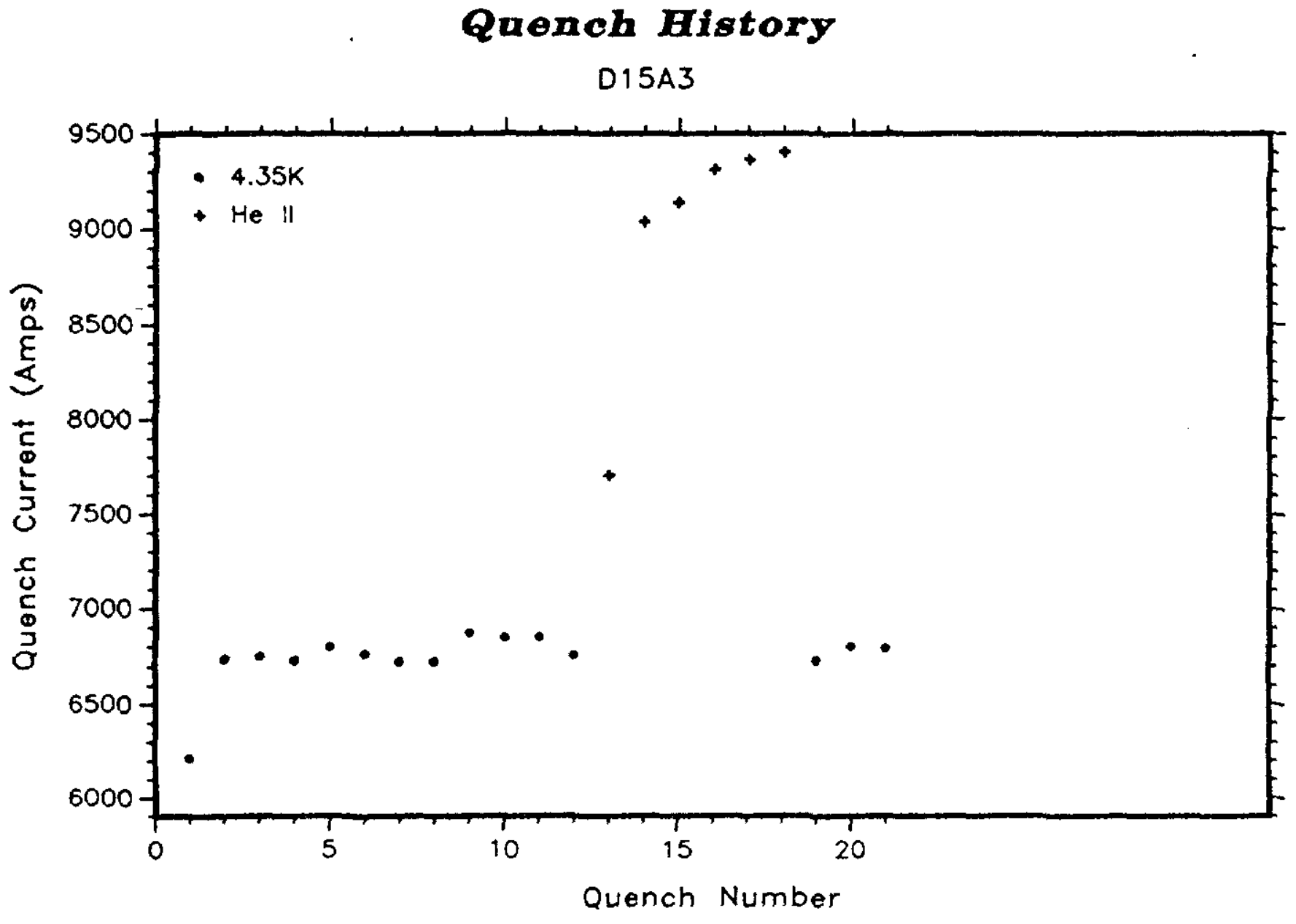

Figure 5 
The evidence is certainly not conclusive on the minimum copper to superconductor ratio. On the basis of Sampson's work, however, a prudent choice would be $1.5: 1$ or higher.

Within the coil package as a whole the current density is affected by the thickness of the insulation. The Tevatron used a 7/12 lap 1 mil Kapton wrap plus a spiral wrap of $7 \mathrm{mil} \mathrm{B-stage/fiberglass} \mathrm{tape} \mathrm{for} \mathrm{a}$ total of about 9 mils of insulation. A new thinner insulation system was developed for the Fermilab low-beta quad program. It consists of a 2 mil Kapton butt lap followed by a 1 mil Kapton butt lap. The outer layer has a $0.1 \mathrm{mil}$ coating of B-stage epoxy (i.e. there is no fiberglass tape). If a cable of Tevatron dimensions is insulated with these two schemes, a coil current density increase of $9 \%$ is realized. It should be noted that a certain uneasiness exists about leaving out the glass tape because of the resulting loss of helium flow channels and helium volume within the coil. It is not understood how sensitive the coil stability (under ramping or beam loss) is to this displaced helium. Tests of the low-beta quad models are now under way which use this insulation scheme.

\section{Coil Loading}

Once every advantage of current density has been taken, the next task is to stably control the large magnetic forces within the coil structure. The coil must be fully supported to resist motion to the highest current. Since the forces increase as the square of the field this becomes rapidly more difficult as the field requirement goes up.

The coil is subjected to a preload at the time of collaring. This preload is successively reduced by creep, by the cooldown and by powering. The preload loss during cooldown results from the slightly faster shrinkage of the coil relative to the stainless steel support collars. Once the coil is powered the turns near the pole are unloaded by the Lorentz force pulling them toward the parting plane. Finally, the radial magnetic forces which increase toward the parting plane react against the support collars resulting in coil distortion. This distortion enlarges the coil cavity further reducing the preload.

It is necessary to examine each of these sources of coil load relaxation to determine if a design can be made that will ensure that the preload to 8.8 tesla can be sustained.

It is important to achieve the highest possible preload on the coils at collaring time in anticipation of the subsequent losses. The main limitation to preload is turn to turn shorts in the coil as the insulation breaks down under pressure. This limiting pressure is in fact quite high. Tevatron coils have been successfully loaded during collaring to $25 \mathrm{~K}$ psi. Recently tests were made by Carson at Fermilab in which cables with various insulating schemes were pressed in a fixture. Between each pressure step hipot measurements were made between turns to determine the onset of insulation breakdown. At least four hours were allowed at each pressure step for the insulation to flow. The results shown in Fig. 6 suggest that $20 \mathrm{~K}$ psi preload can be safely used with Kapton only. 


\title{
Insulation Breakdown Test
}

\author{
(Data Taken 2/17/88)
}

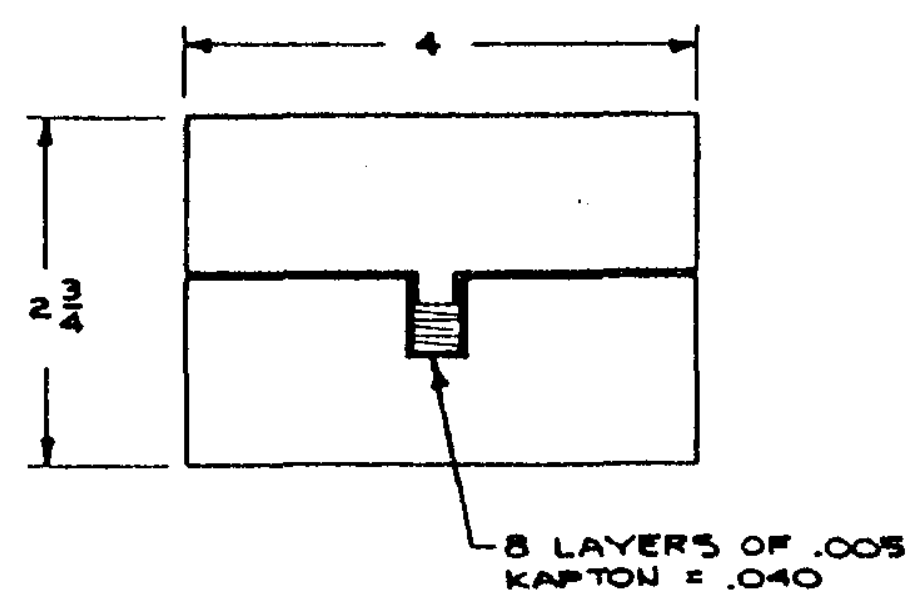

Insulation Scheme

1. One layer of 1 mil kapton, $1 / 2$ lap and one layer 4 mil glass tape $90 \%$

2. One layer of 2 mil kapton butt lap and layer of 1 mil kapton butt lap

3. One layer of 1 mil kapton $3 / 4$ lap
Breakdown

None at $49 \mathrm{~K}$ psi

(limit of the press)
Turn to turn short

at $35 \mathrm{~K}$ psi after

1 hour

Turn to turn short at

$25 \mathrm{~K}$ psi

Figure 6 
It is important to note that the pressure must be uniform across the face of the cable. During the collaring process the collars may be distorted so that stress concentrations may occur at the inner or outer edge of the cable far in excess of the average pressure. The collaring process must therefore be carefully modeled and designed to insure that such stress concentrations do not occur.

Current practice calls for the collars to be locked together with keys, a technique originally used on the Tevatron quadrupoles. The coil is over-compressed, the keys are inserted and the press pressure is removed. Because of the slight gap that remains between the keys and collar the coil relaxes to a preload substantially below the collaring pressure. This is illustrated in Fig. 7 in an early BNL SSC magnet. ${ }^{4}$ A new scheme developed by Taylor at LBL uses tapered keys that are engaged by a separate set of hydraulic cylinders just as the collaring press nears its maximum pressure. The tapered keys pull the collars together as they are forced in and thereby preserve the preload (see Fig. 8). ${ }^{5}$

Once the coil is collared and is under pressure the plastic materials within the coil will begin to creep. Strain gauges have been used to monitor the coil preload through the assembly process. Considerable prestress loss due to creep has been observed on SSC models. Performance loss due to creep, however, has not been observed in Tevatron magnets, even those stored for years at ambient temperature. It is very important to cure the coils at sufficiently high pressure so that most of the soft materials flow away from contact surfaces and the remaining material has taken up most of its creep.

The next major loss of preload results from shrinkage of the coil relative to stainless steel collars. In principle, aluminum collars shrink more than the coil and should load the coil during cooldown:

\author{
Shrinkage to $4 \mathrm{~K}$ \\ Coil $3.3 \mathrm{mils} / \mathrm{inch}$ \\ Stainless steel $\sim 3 \mathrm{mils} /$ inch \\ Aluminum 4 mils $/$ inch
}

In practice this additional preload is not observed. The preload at room temperature, however, seems to be preserved. It is, therefore, still attractive to use aluminum collars. Aluminum collars have the additional feature that they have much better thermal conductivity than those of stainless steel.

The remainder of the preload loss occurs during powering. It results from the azimuthal component of the Lorentz force pulling the coil away from the pole and from the enlargement of the coil cavity because of collar distortion. If the pressure on the coil at the pole goes to zero the coil will become unstable and will not sustain high current. 
AVG. INNER \& OUTER (

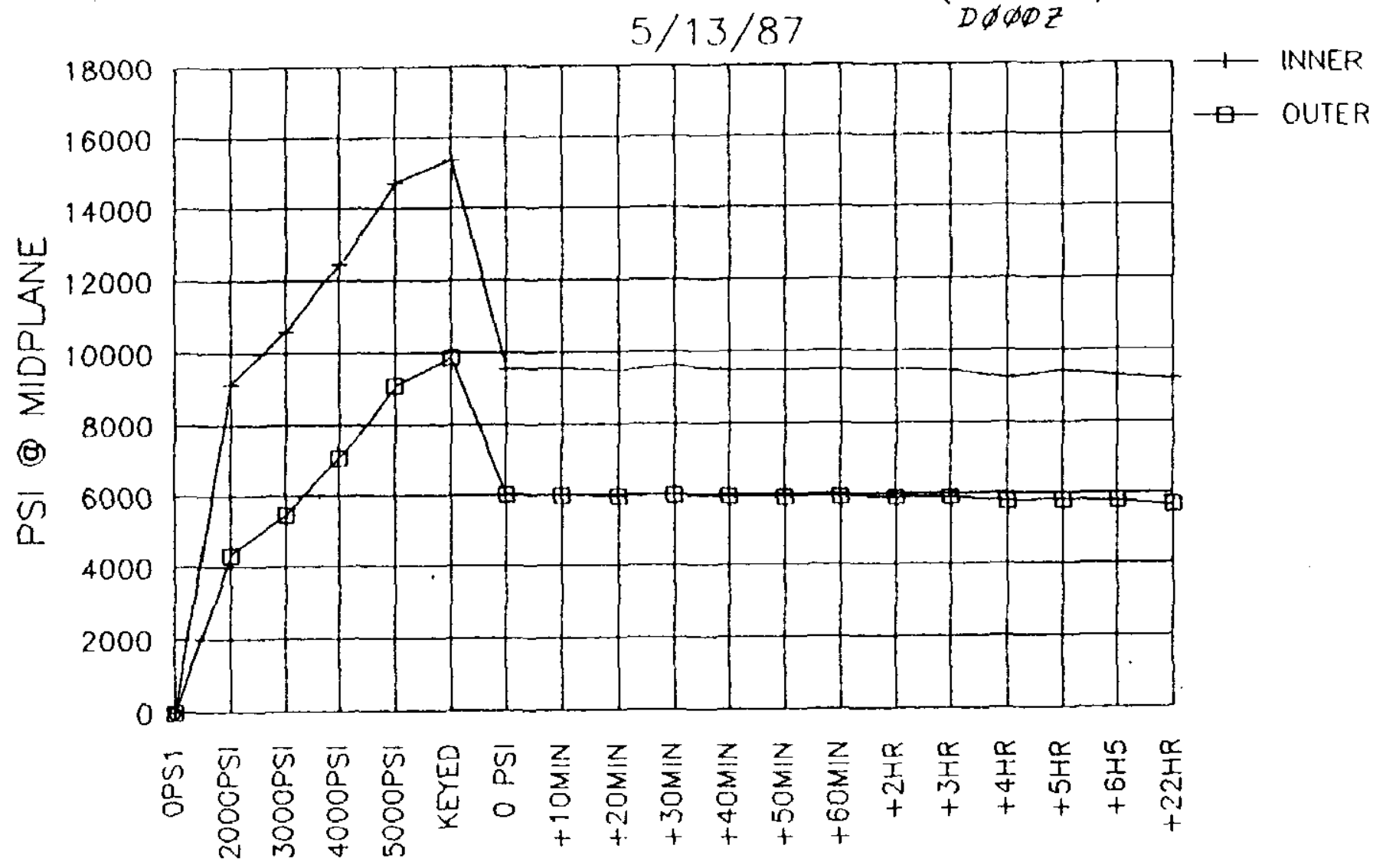

COIL LOADING HISTORY

Figure 7

Prestress Loss on an Early SSC Magnet 


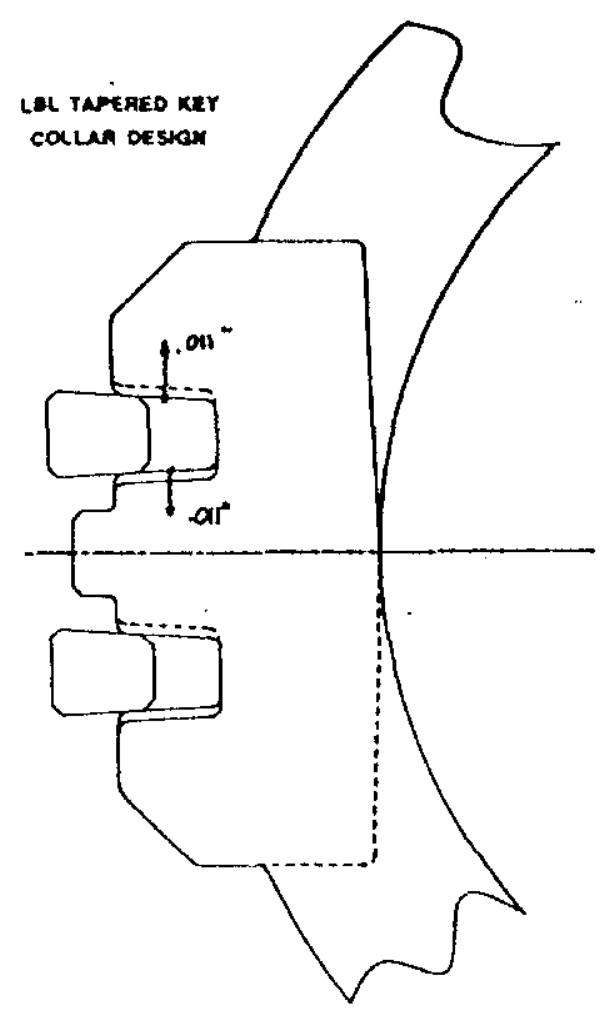

KEY ENGAGEMENT

\section{COIL STRESS AKO KEY FORCE FOR MODEL DI $5 A-3$}

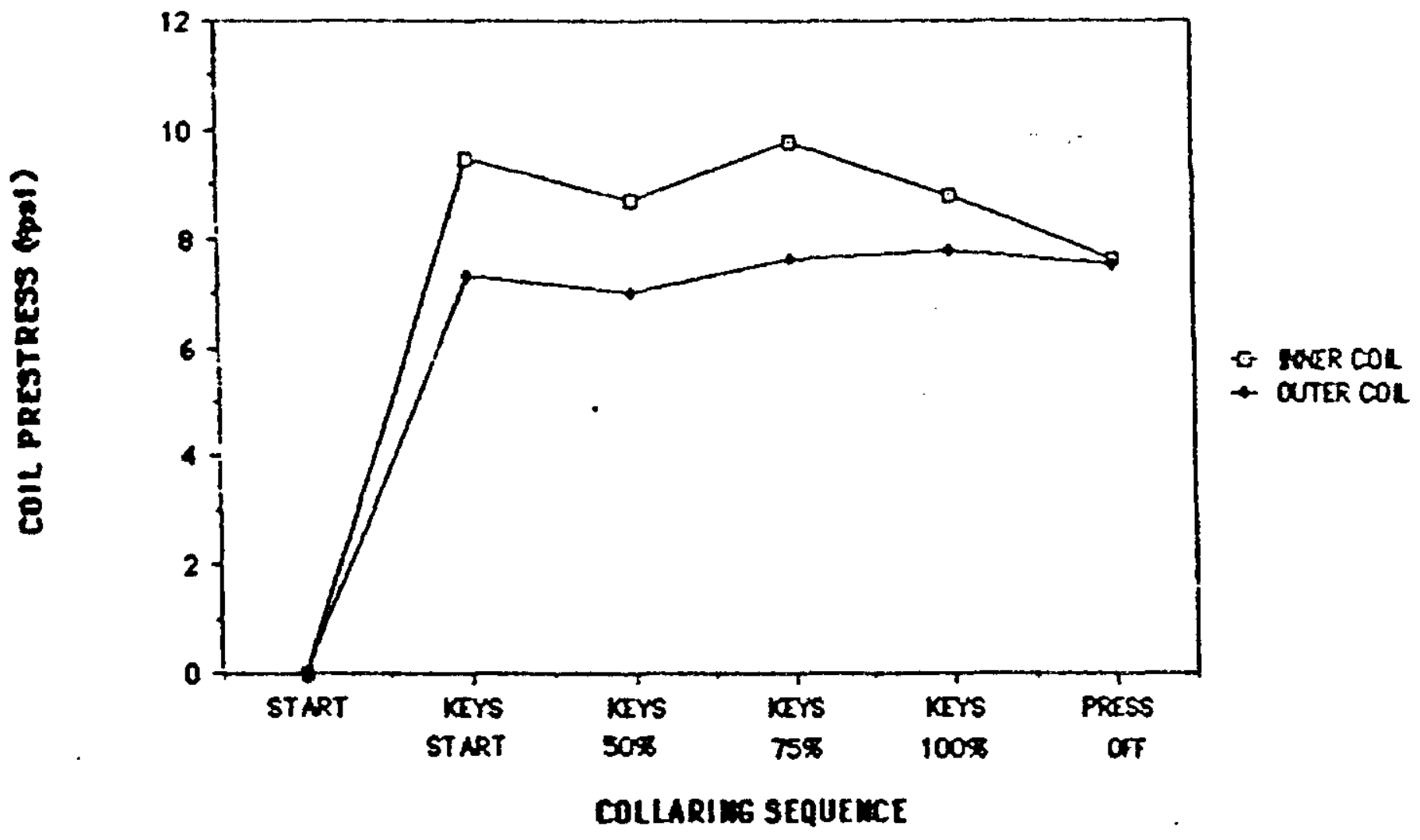

Figure 8

Collaring Prestress Loss Using Tapered Keys 
The azimuthal Lorentz force acts on the individual turns relaxing the pressure on those near the pole and compressing those near the parting plane. This azimuthal pressure increases nearly linearly from zero at the parting plane to a maximum at the pole turn given by the product of the field at that point and the current. Figure 9 shows the azimuthal pressure as a function of turn number for the SSC coil.

Consider a simple spring model for the coil in which the total azimuthal Lorentz force is concentrated at the center of the opening spring.

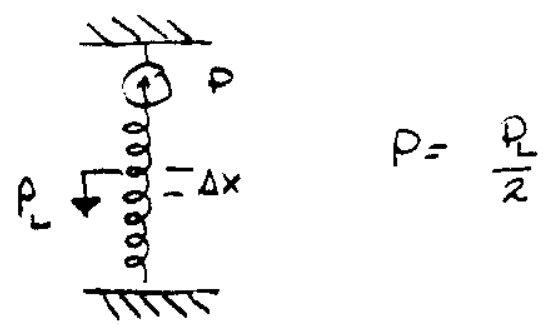

In this model the coil pressure reduction at the pole is half of the total Lorentz force. In a more complex model due to Tollestrup the actual force on each turn is considered. In this model the fraction of the total azimuthal Lorentz force felt at the pole is about two-thirds. Even this model may not adequately describe the pressure distribution in the coil because it ignores friction between the coils and the collars. It also ignores the fact that the spring constant (modulus) is different in compression than in tension. In particular, the coil exhibits a higher modulus when it is being relaxed than when it is being compressed. Consider the simple spring model but now make the modulus higher for the turns relaxing near the pole than those being compressed near the parting plane:

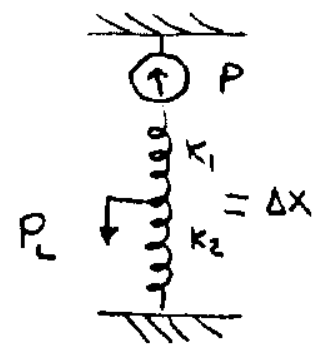

$$
\begin{aligned}
& P=k_{1} \Delta x \\
& P_{L}=k_{1} \Delta x+k_{2} \Delta x \\
& P=\frac{k_{1}}{k_{1}+k_{2}} P_{L}
\end{aligned}
$$

This refinement would allow the fraction of the total Lorentz force seen at the pole to be even greater than two-thirds. For the purposes of the discussion, however, a value of two-thirds will be assumed.

The other Lorentz force driven preload relaxation results from the enlargement of the coil cavity due to distortion of the collars. Since collars, and aluminum collars in particular, will flex to some degree it will be important to utilize the iron yoke for additional strength. The steel shrinks less than the aluminum during cooldown so there must be some mechanism to keep the collars in intimate contact with the yoke and to make the yoke itself resistant to flexing. One approach is shown in Fig. 10. $\mathrm{A}$ coil design for the LHC uses an aluminum outer skin that pulls the yoke halves down against the collars at cooldown.

Since the beginning of the SSC dipole program, substantial effort has gone into instruments to study the performance of these magnets 

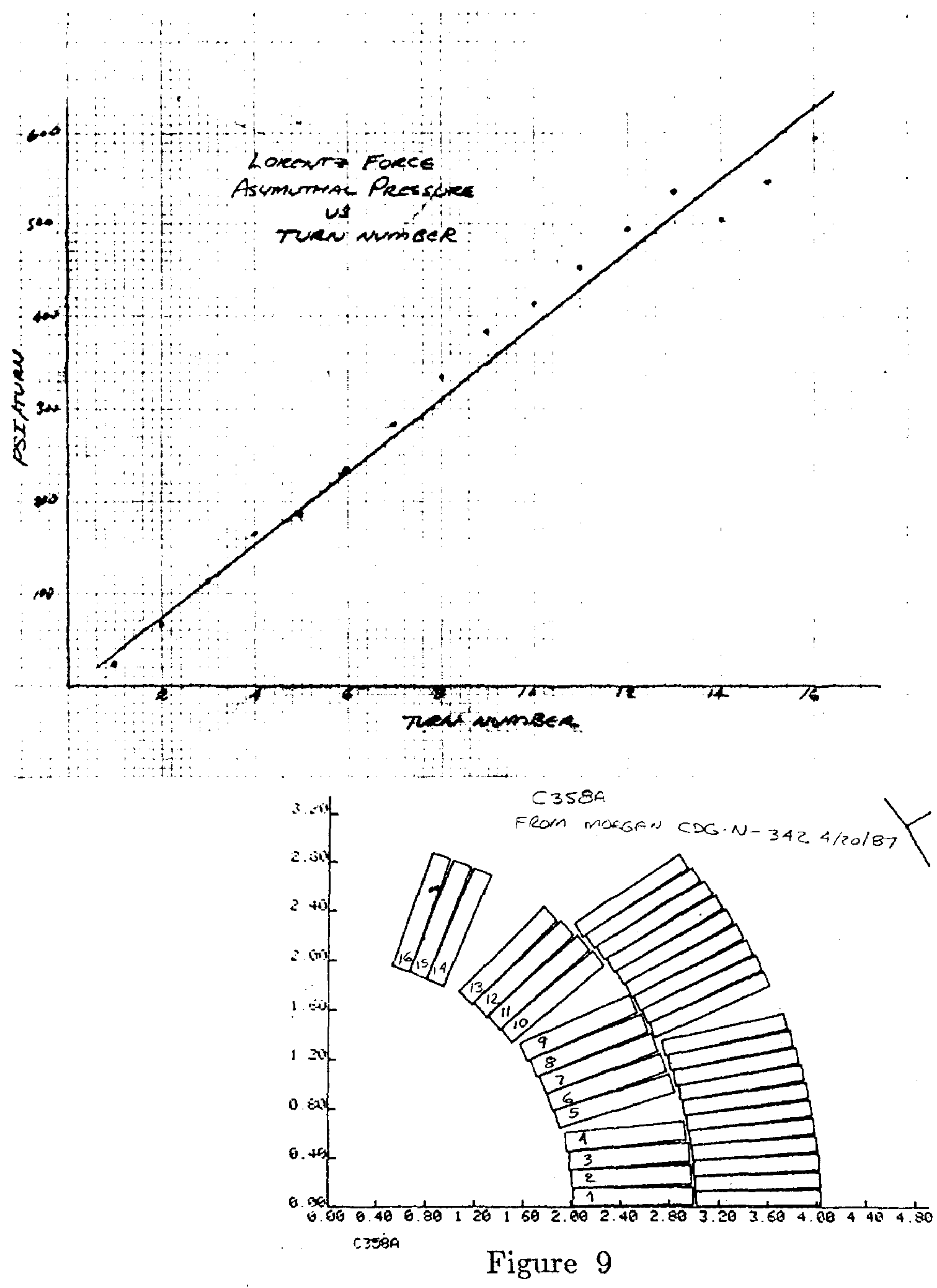

Lorentz Force Coil Loading 

under power. In particular, strain gauge techniques have been improved to the point where they give believable results. Strain gauges are attached to the poles of the coils as shown in Fig. 11 . Figures 12 and 13 show some typical curves of stress at the pole as a function of the square of the coil current. The stress is almost, but not exactly proportional to the current squared.

Figure 12 is particularly interesting because it involves a BNL SSC 1.5 meter model (DSS6) that was first tested in the normal configuration where the collars alone support the coil. The model was then modified by putting shims between the collared coil and the yoke and leaving a $10 \mathrm{mil}$ gap between the yoke halves at the parting plane (Fig. 14). ${ }^{7}$ The shims firmly press the collars against the yoke which results in less collar flexing and the magnetic attraction between the yoke halves actually increases the coil load with increasing current. The net effect is that the coil in the shimmed magnet unloads much more slowly with current. The same shim arrangement was used in SSC magnet DD0012 whose stress curve is shown in Fig. 13. Note that high coil preload is maintained out to the highest current (about 7600 amps). Magnet DD0012 was the best performance of any SSC magnet so far. Figure 15 shows the quench history of DD0012 compared to the next best magnet, D000X.

It is possible to gain some understanding of coil unloading by comparing the curves in Figs. 12 and 13 with the spring models described earlier. Figure 16 shows the contribution to the pressure at the pole of the inner coil from the coil azimuthal Lorentz force, the yoke gap and the collar defection. The stress gauge reading at the pole starts with the prestress, $P_{0}$ at zero current and is increased or decreased by the different contributions of the magnetic force. Each of the pressures should be proportional to the square of the current. The strain gauge reading should therefore be:

$$
\begin{aligned}
\mathrm{P}_{\mathrm{SG}} & =\mathrm{P}_{0}-\mathrm{P}_{1}+\mathrm{P}_{2}-\mathrm{P}_{3} \\
& =\mathrm{P}_{0}+\alpha \cdot \mathrm{I}^{2}+\beta \mathrm{I}^{2}+\gamma \mathrm{I}^{2} \\
& =\mathrm{P}_{0}+(\alpha+\beta+\gamma) \mathrm{I}^{2}
\end{aligned}
$$

where

$$
\begin{aligned}
& P_{S G} \quad-\text { strain gauge pressure } \\
& P_{1} \quad-\text { pressure due to coil azimuthal Lorentz force } \\
& P_{2} \quad-\text { pressure on the coil due to the yoke gap } \\
& P_{3} \quad-\text { pressure due to collar distortion }
\end{aligned}
$$

The slope of the stress curves is the sum of $\alpha, \beta$ and $\gamma$.

Consider the three pressures one at a time. 


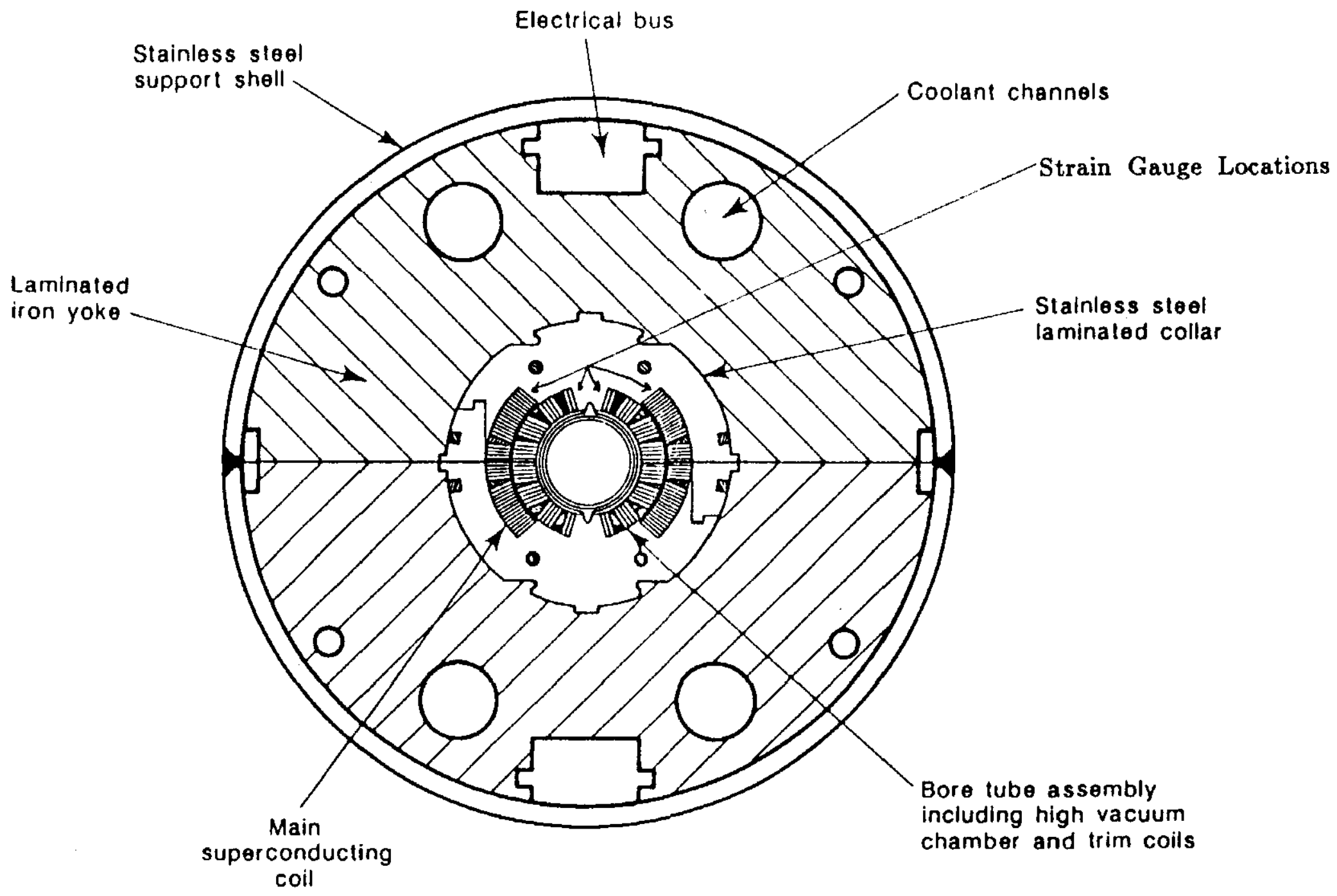

Figure 11

Position of Strain Gauges for the SSC Coil 


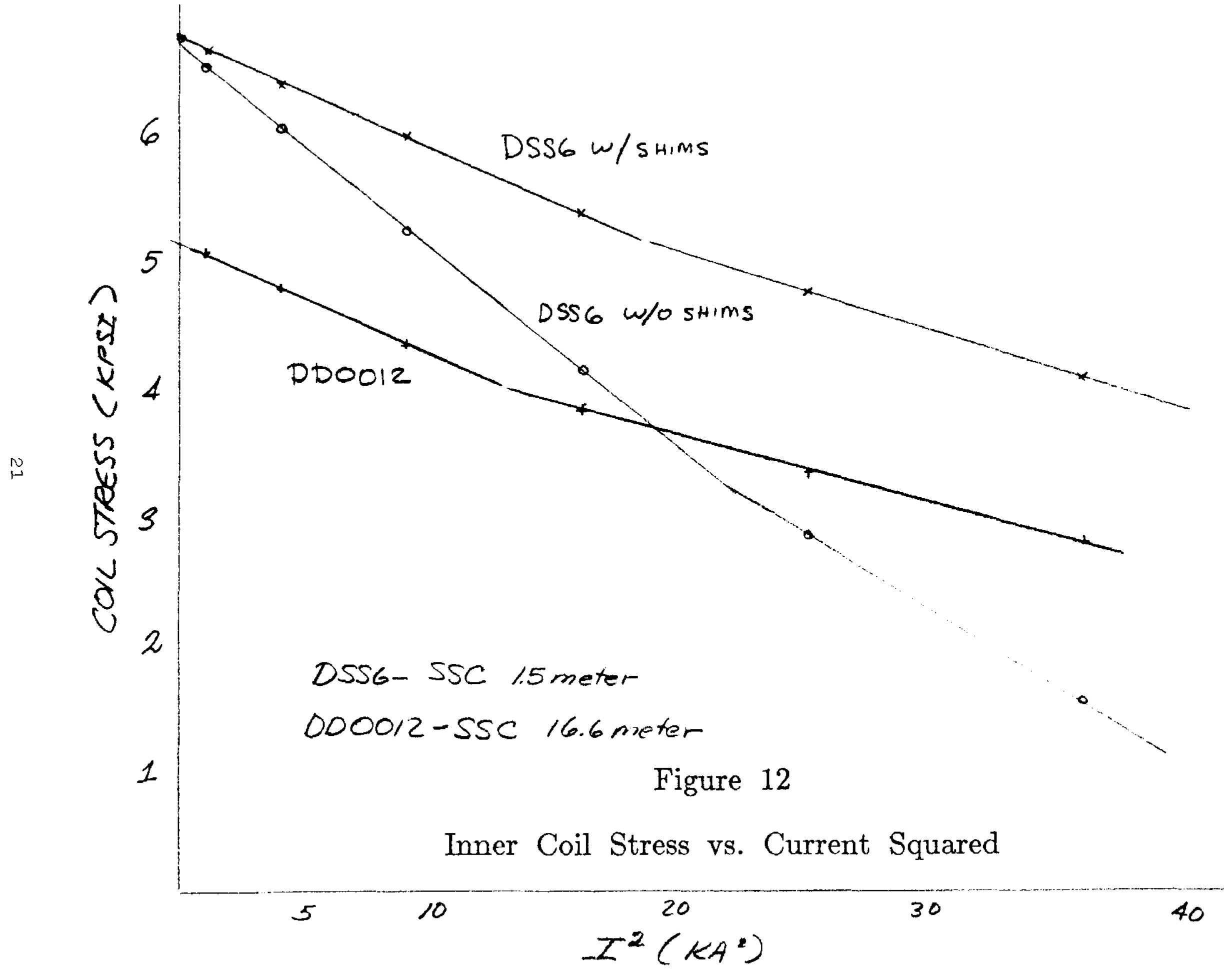




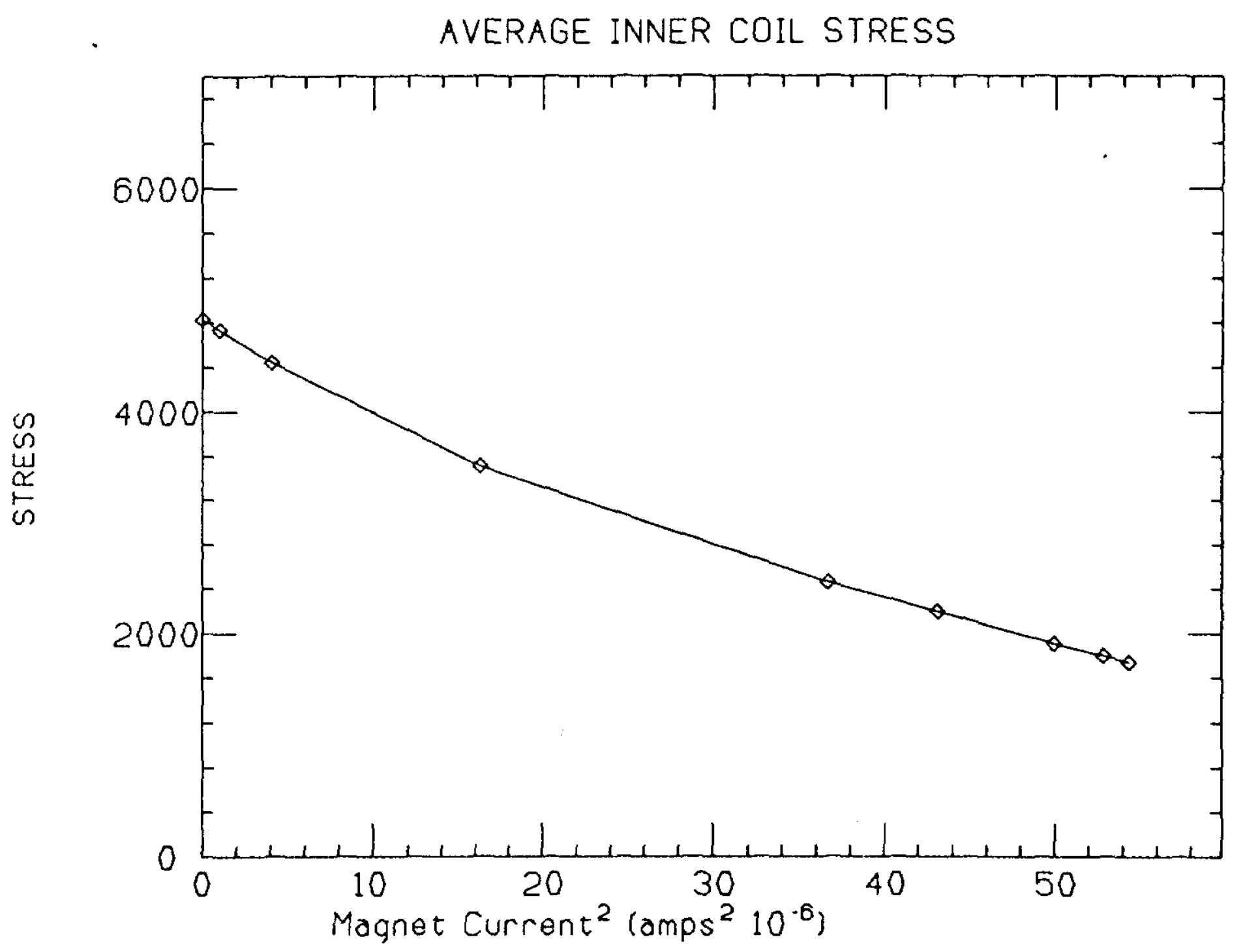

Figure 13

Inner Coil Stress on SSC Magnet DD0012 


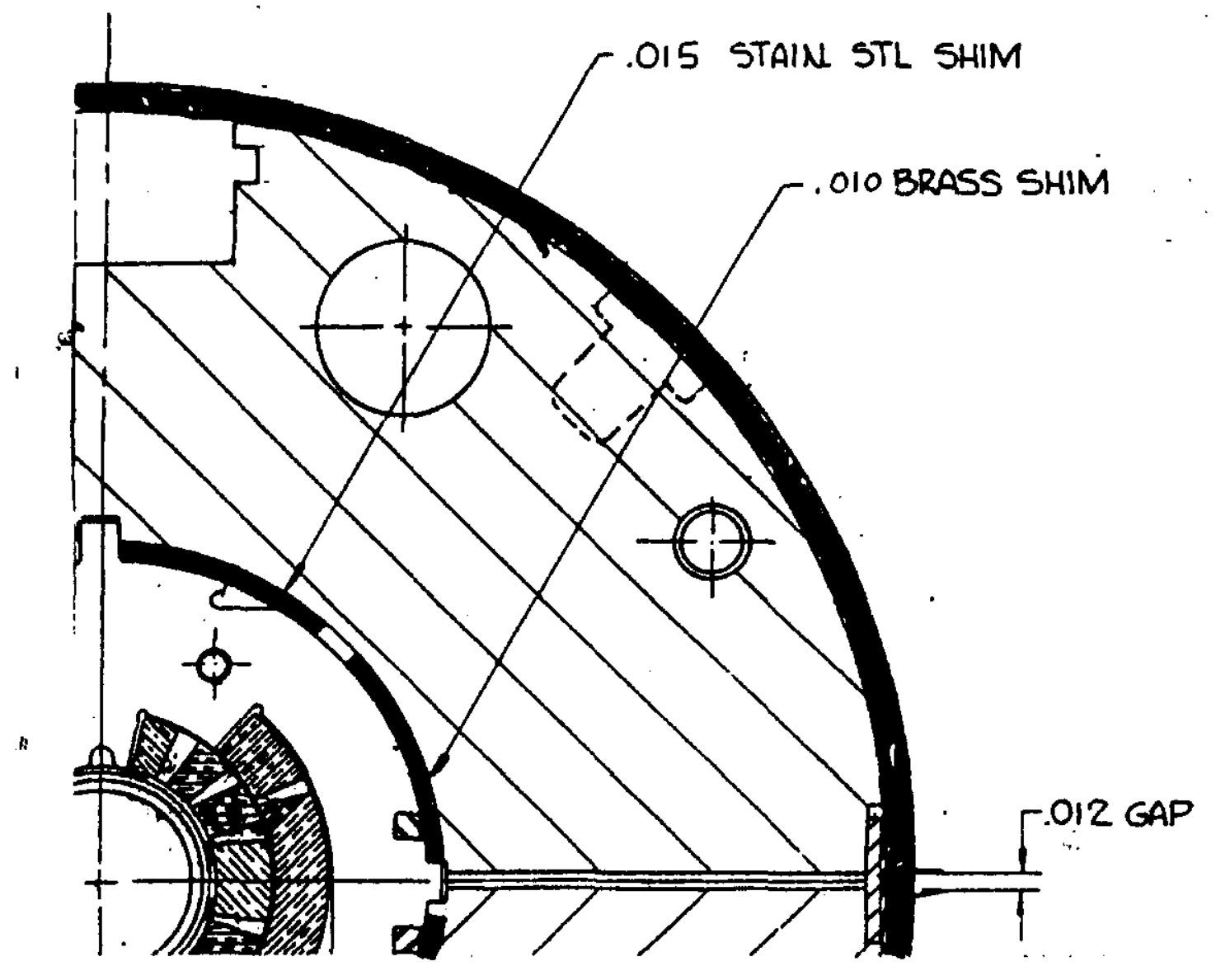

Figure 14

Shim Location in Magnets DSS6 and DD0012 


\section{Quench History}

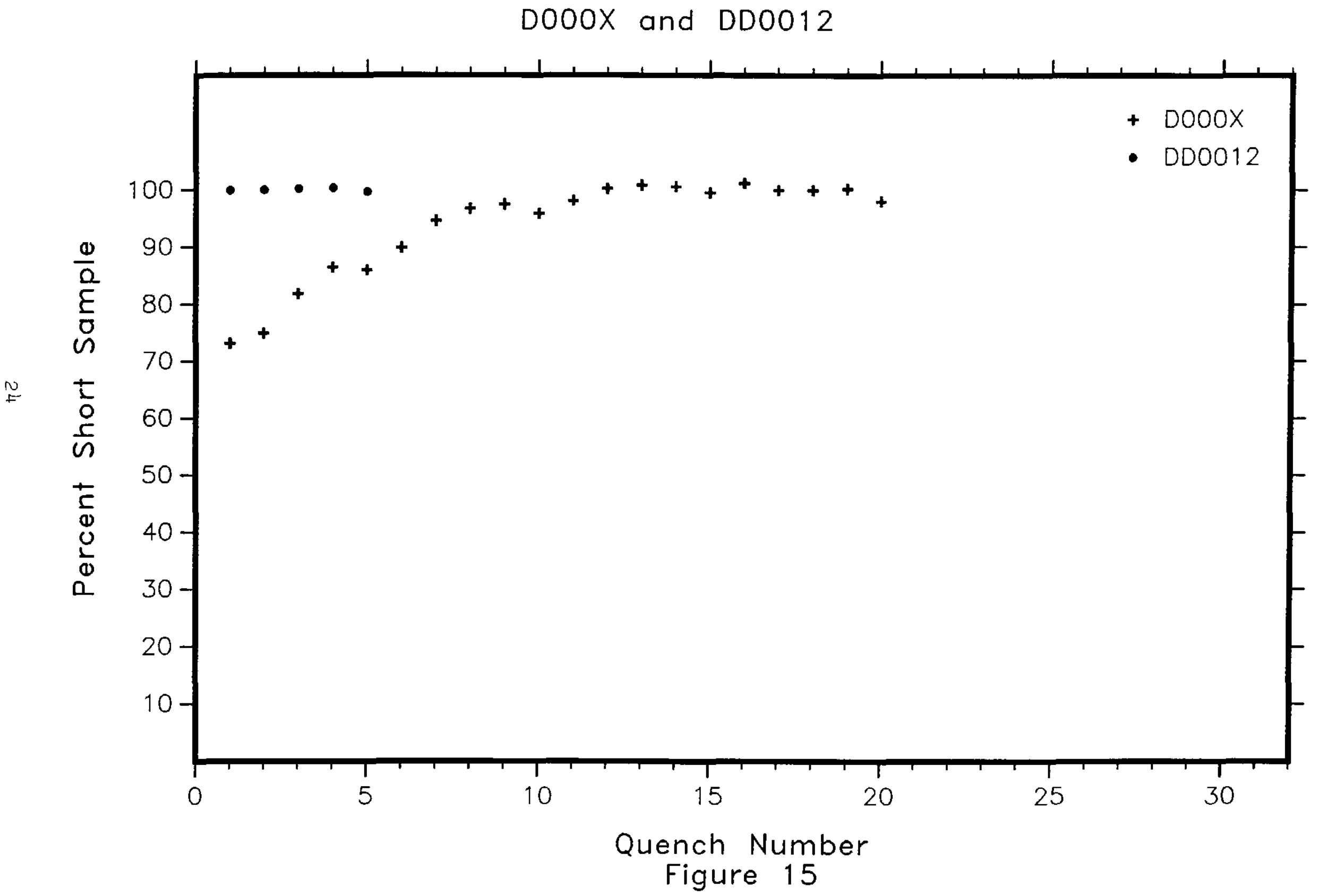




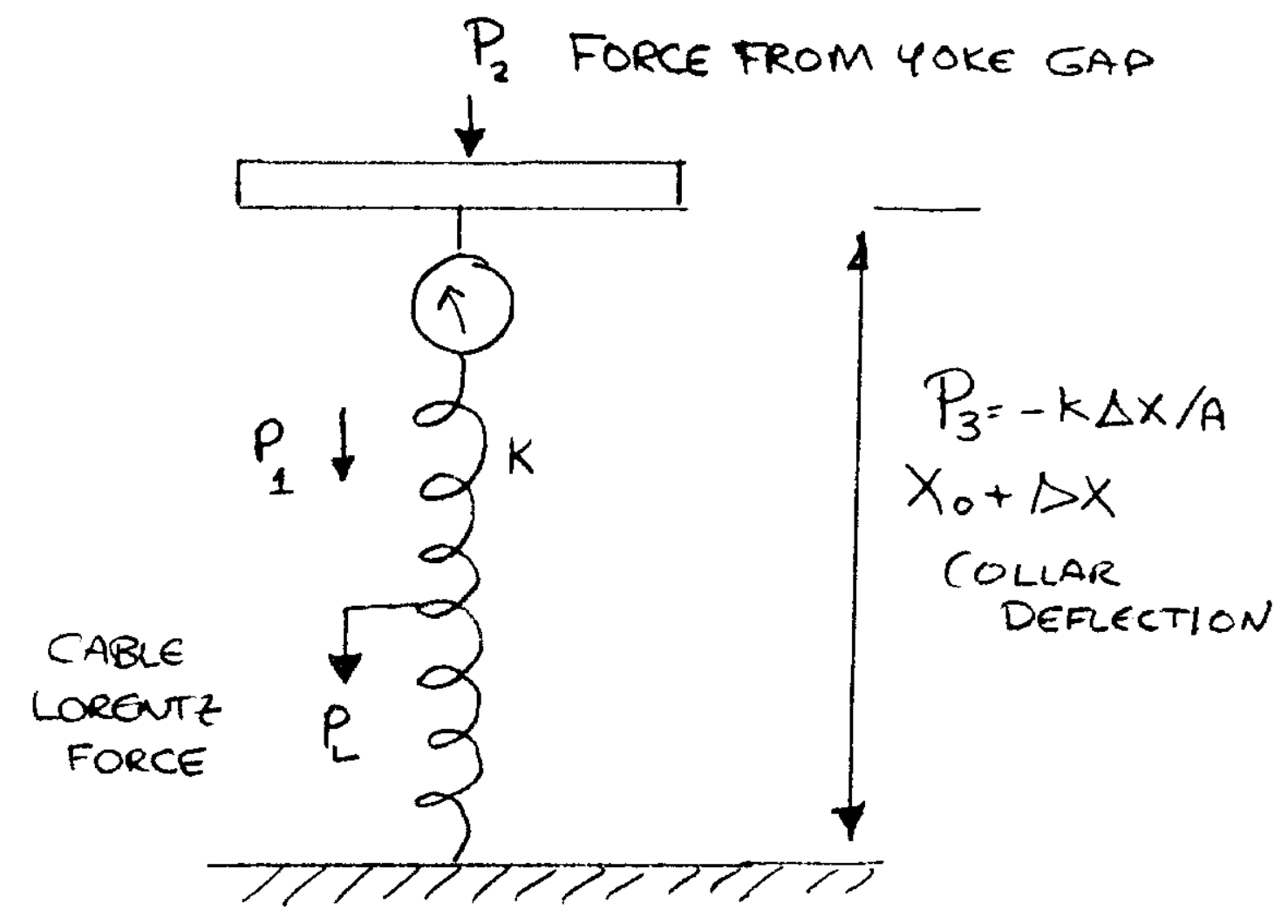

$$
P_{S G}=P_{0}-P_{1}+P_{2}-P_{3}
$$

Po - Azimuthal Prestress

$P_{1}$ - Azimuthal Component of coil Lorentz Force

$P_{2}$ - Pressure on the coil due to the presence of the yoke gap

$P_{3}$ - Pressure relaxation due to collar distortion

$P_{S G}$ - Strain gauge reading

Figure 16

Coil Loading Model

25 
The total inner coil azimuthal Lorentz force has been calculated by Morgan for the SSC cross section at operating current $(6.6 \mathrm{~K} \mathrm{amp})$ to be 5820 psi. If the stress at the pole is two-thirds of the total (Tollestrup model) the contribution to the slope from the azimuthal Lorentz force can be predicted:

$$
\alpha=\frac{2 / 3 * 5820}{(6.6)^{2}}=-89 \mathrm{psi} / \mathrm{KA}^{2}
$$

The pressure on the coil resulting from the presence of the gap between the yoke halves has been calculated by Morgan for this particular magnet to be $1750 \mathrm{psi}$ at $6.6 \mathrm{KA}$.

$$
\beta=\frac{1750}{(6.6)^{2}}=+40.2 \frac{\mathrm{psi}}{\mathrm{KA}^{2}}
$$

The effect of this pressure is to decrease the slope.

Calculation of the deflection of the collars is a complicated undertaking using finite element analysis. The results are compromised by an incomplete understanding of the properties of the coil. A calculation at BNL for this magnet shows that the collars flex radially by about 2 mils at the parting plane. This gives rise to a small increase in the azimuthal size of the coil and a resulting loss of $1000 \mathrm{psi}$ in coil loading.

$$
\gamma=\frac{1000}{(6.6)^{2}}=-23.0 \mathrm{psi} / \mathrm{KA}^{2}
$$

These numbers taken together should give a prediction for the two slopes shown in Fig. 12 for short model magnet DSS6.

$$
\begin{aligned}
& \text { DSS6 - without shims } \\
& \begin{array}{ll}
\alpha & =-89 \mathrm{psi} / \mathrm{KA}^{2} \\
\beta & =0 \text { (There is no yoke gap) } \\
\gamma & =-23.0 \mathrm{psi} / \mathrm{KA}^{2}
\end{array} \\
& \text { Slope }=-89-23=-111 \mathrm{psi} / \mathrm{KA}^{2} \\
& \text { DSS6 - with shims } \\
& a=-89 \mathrm{pis} / \mathrm{KA}^{2} \\
& \beta=+40.2 \\
& \gamma=\begin{array}{l}
0 \text { (The coil is backed up by the yoke and should not } \\
\text { deflect) }
\end{array} \\
& \text { Slope }=-48.8 \mathrm{psi} / \mathrm{KA}^{2}
\end{aligned}
$$


In comparing these results to the data, note that the curves do not have a constant slope. A careful look at the DD0012 curve shows that there are two fairly distinct slopes, one for low currents and a shallower one at high currents. The results can then be tabulated as follows:

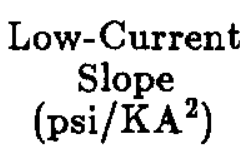

DSS6 (no shims)

DSS6 (shims)

DD0012 (shims)
$-166.0$

$-87.6$

$-79.6$
High-Current

Slope

$\left(\mathrm{psi} / \mathrm{KA}^{2}\right)$

$-118.0$

$-59.1$

$-52.9$
Predicted

Slope

$\left(\mathrm{psi} / \mathrm{KA}^{2}\right)$

$-111.0$

$-48.8$

$-48.8$

Within the limits of uncertainty of the model the agreement at high current is quite reasonable. The steeper slope in the case of the shimmed magnets is most likely the result of the collars not yet being up against the yoke. At high current the collars are firmly against the yoke. The change in slope of the unshimmed magnet is difficult to explain.

This exercise then provides the basis for estimating the stress loss under power. The magnets above have the yoke gap which is probably not practical in a real magnet. With the collaring structure rigidly constrained and no yoke gap, the inner coil stress loss due to the azimuthal Lorentz force can be estimated for similar cross sections as follows:

Azimuthal Lorentz pressure at pole turn $=$

$$
f\left(\frac{B_{\max } \times I_{o}}{2}\right) \frac{n}{w}
$$

where:

$f$ is the fraction of the total azimuthal pressure seen at the pole $\operatorname{turn}(2 / 3$ is assumed)

$\mathrm{B}_{\max }$ is the high-field point at the inner pole turn

$I_{0} \quad$ is the current

$n$ is the number of turns in the quadrant

w is the width of the conductor 
If one uses the various schemes mentioned earlier to preclude the loss of preload this formula can be used to estimate the amount of preload required for a magnet of given strength.

Since the preload on high-field magnets is critically important, the coils must be built with sufficient precision that the preload is uniform along the length. The dimensional tolerance on the conductor must be very tight. Carson at Fermilab has developed a measuring machine that measures cable, on-line at operating stress, to better than \pm 0.0001 inch. With this machine supplied to the vendor it is possible to set a cable thickness specification at $\$ 0.0002$ inch and get cable within those limits. The tooling must also have good precision. Recent coils at Fermilab have been made with variations in the thickness of less than \pm .001 inch. This compares to \pm .002 inch for the Tevatron.

Ends have traditionally been a weak part of superconducting magnets. The complex geometry of the saddle end with spacers to adjust the end field is hard to support adequately. The transition from the straight section to the end turns is also a source of high-stress points that can lead to turn to turn shorts. Study is under way at Fermilab to design an end that is free of high-stress points and in which the end turns are properly supported. In the constant perimeter end the conductors take nearly their natural shape. Analytical work has been done on this problem a number of years ago at Rutherford Lab and more recently at Argonne Lab. Using this work and the CAD system greatly improved ends can be made. Once the design is complete the complex support pieces can be automatically machined from the CAD information. Figure 17 shows some of the drawings made in the CAD during the design process.

\section{An 8.8 Tesla Dipole Design}

First attempts have been made by Riddiford to design a coil cross section to meet the specification shown in Table I. The magnet is shown in Fig. 18. It makes use of the conductor developed for the lowbeta quad system. The load line and cable characteristics are shown in Fig. 19. At least according to this graph the magnet can reach $10 \mathrm{~T}$ at $1.8 \mathrm{~K}$ using $\mathrm{NbTiTa}$ conductor.

It is necessary now to estimate the preload needed to ensure adequate loading through the operating range. Including operating margin the magnet should go to 10 tesla. If it is assumed that no preload is lost except during powering, we can calculate the loss in azimuthal preload on the inner coil:

$$
\Sigma \mathrm{P}_{\theta}=\left(\frac{\mathrm{B}_{\max } \cdot \mathrm{I}_{\mathrm{o}}}{2}\right) \frac{\mathrm{n}}{\mathrm{w}}=28.0 \mathrm{~K} \mathrm{psi}
$$

If the pole turn sees two-thirds of this, the expected preload loss in the inner coil would be $18.7 \mathrm{~K}$ psi. In order to account for other possible losses, a preload of at least $20 \mathrm{~K}$ psi is necessary. This high load requires that considerable attention be paid to the insulation 

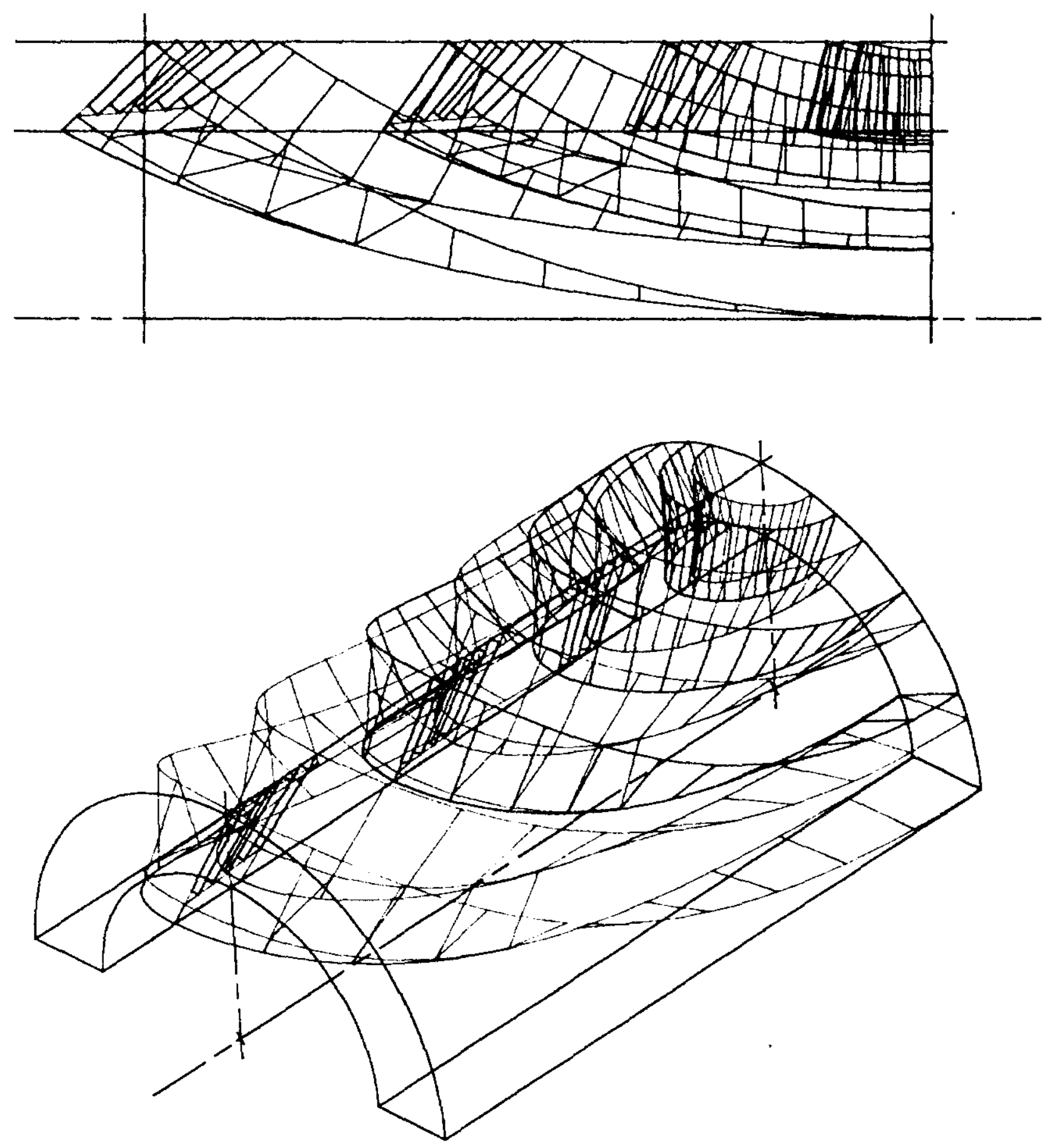

\section{Figure 17}

CAD Drawings of End Turn Design 


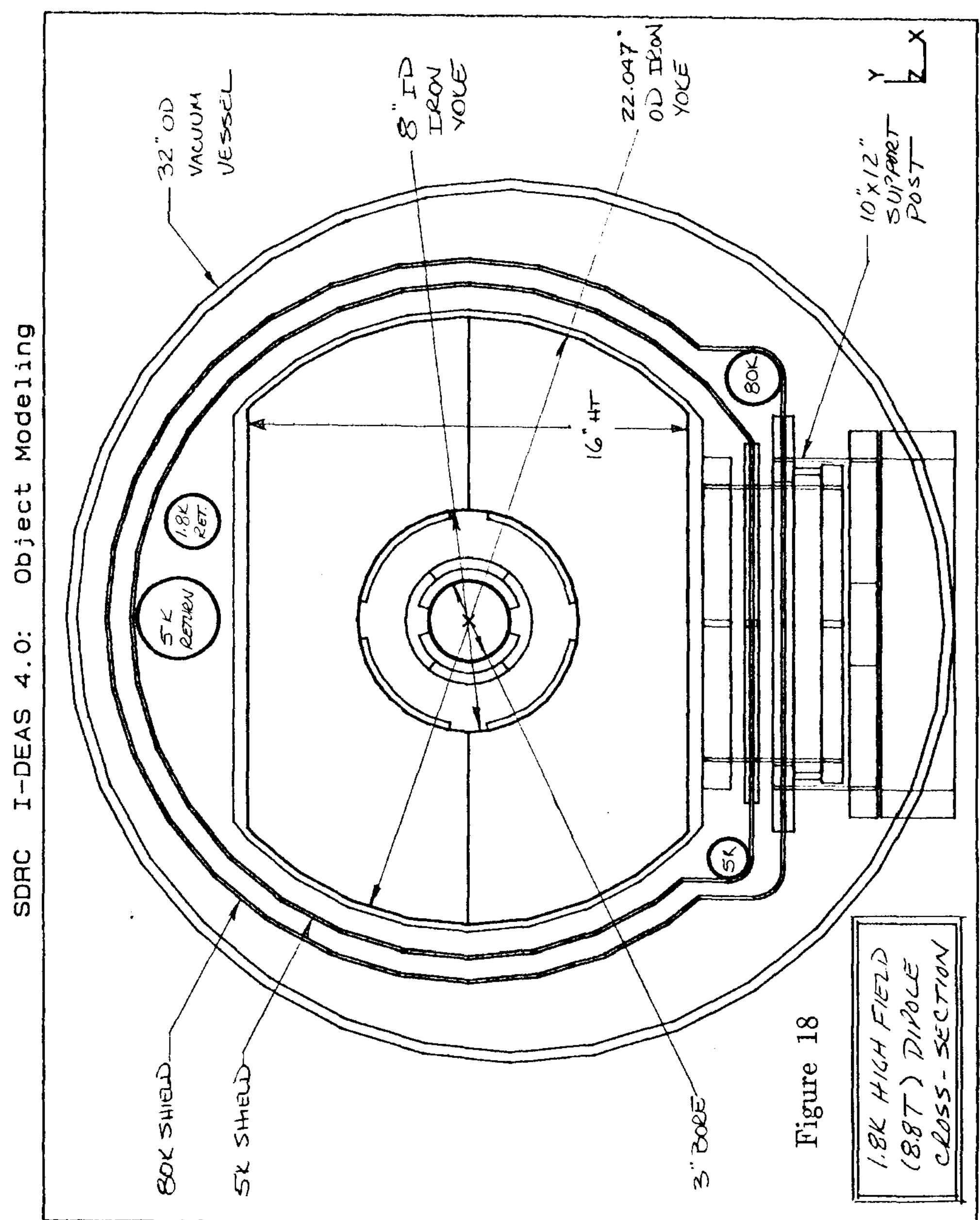




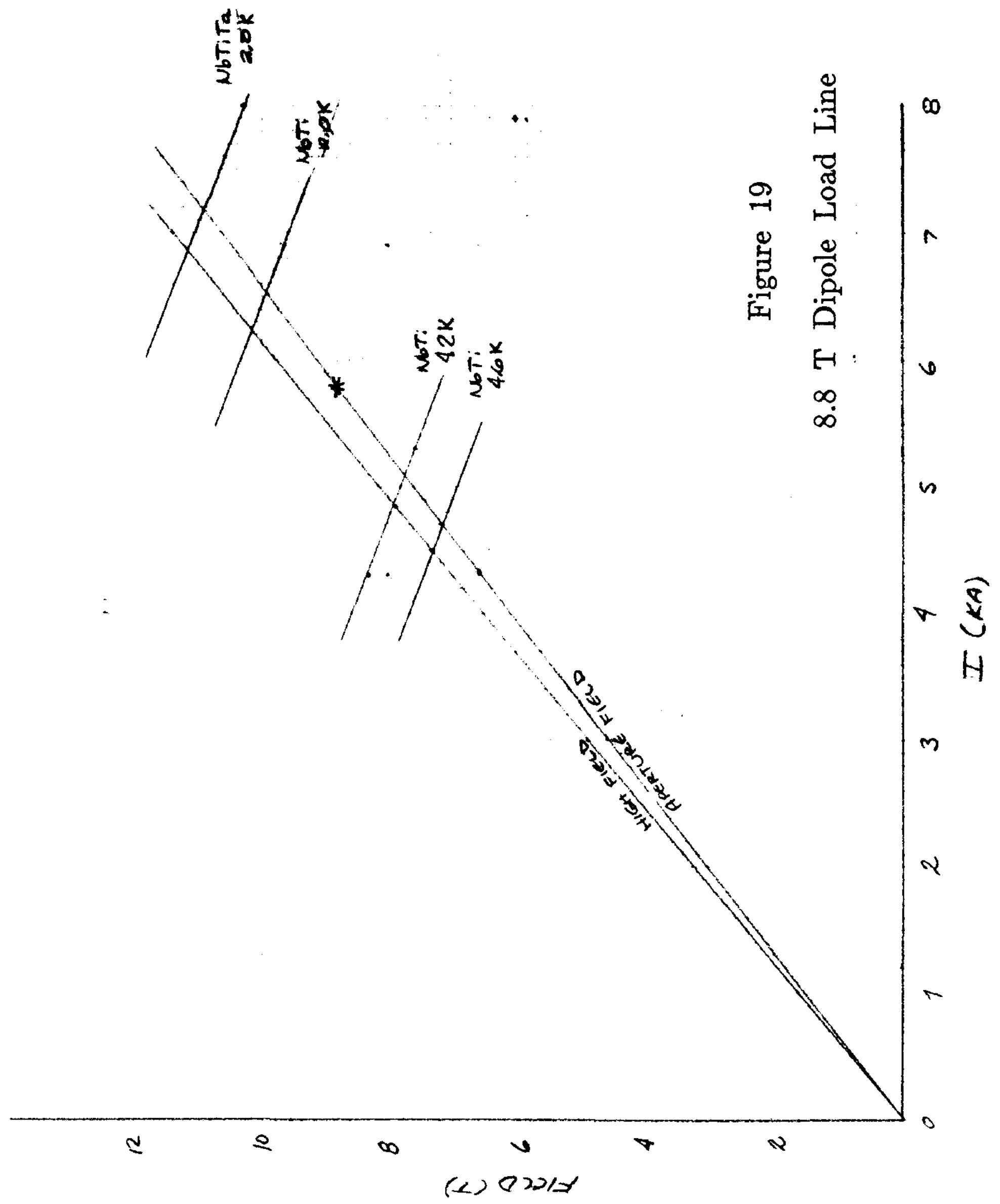


system. If this preload can be obtained and the integrity of the insulation scheme can be demonstrated, an 8.8 tesla magnet can probably be successfully made.

State of the Art in High-Field Accelerator Magnets

It is worthwhile to look at the performance of recent high-field magnets and compare design features with those of the proposed 8.8 tesla magnet. Five magnets will be compared: Tevatron, SSC, HERA, LHC Model and the Tevatron $8.8 \mathrm{~T}$.

A 1 meter LHC model has recently been completed and tested by CERN and Anzaldo. Its specification is shown in Fig. 20 and a load line in Fig. 21. ${ }^{8}$ Although exact numbers are not available the magnet is said to have trained to 9.1 tesla in about three quenches.

HERA magnets also demonstrate excellent performance. Figure 22 shows that for among three full size (9 meter) magnets there was only one training quench. The magnets performed well beyond their nominal operating current.

As shown in Fig. 5, SSC 1 meter models have operated at 9.4 tesla.

Table III compares these magnets at operating current and maximum current (short sample). The total inner coil azimuthal Lorentz force pressure is estimated in each case to show the level of stress in the coil.

In terms of coil stress level the HERA magnet and the LHC model operate at about the same level because of the larger aperture of the HERA magnet. The coil stress of the proposed $8.8 \mathrm{~T}$ magnet is nearly twice that of the LHC and HERA!

\section{Summary}

In order to realize a viable 8.8 tesla dipole design, the following are essential:

1) High-current density

2) A very rigid mechanical support for the coil

3) Coils of high precision and uniformity

4) High coil prestress sustained through collaring, cooldown and powering

5) An insulation scheme able to maintain its integrity at these high stresses 


\title{
Specification of
}

\author{
CERN/Ansaldo
}

\author{
LHC $1.5 \mathrm{~m}$ Model
}
1) Central field $B_{0}$
$8.0 \mathrm{~T}$
2) Peak field at $B_{0}$
$8.5 \mathrm{~T}$
3) Current at $B_{0}$
9140 amps
4) Inner coil diameter
$50 \mathrm{~mm}$
5) Stored energy
$160 \mathrm{KJ}$
6) Cable geometry
$1.3,1.67 \times 12.6 \mathrm{~mm}^{2}$
7) $\mathrm{Cu} / \mathrm{SC}$ ratio
1.8:1

Figure 20 
$I[A]$

Figure 21

Load Line for LHC Model

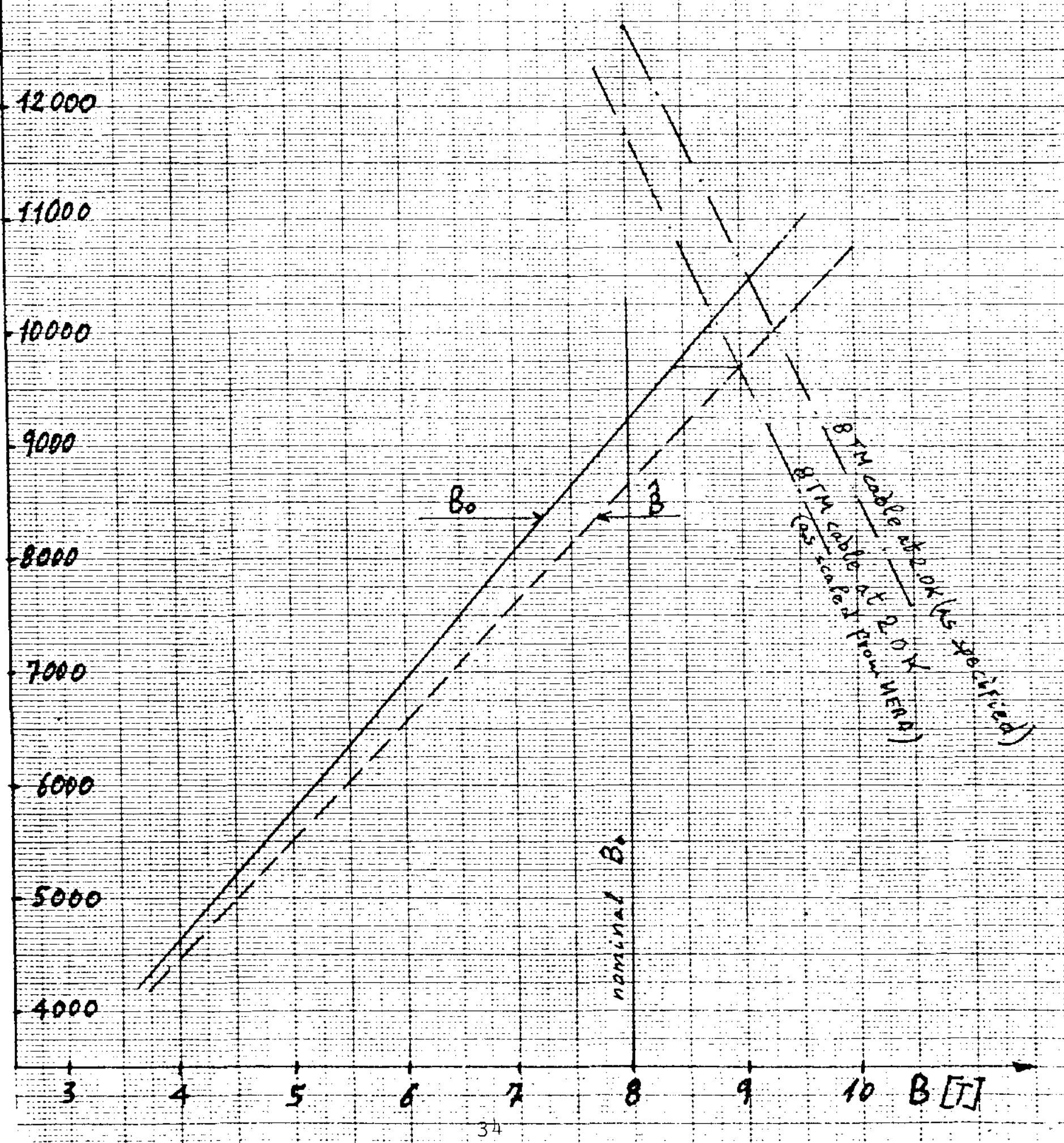


Quench History

HERA Dipoles $9 s 3,9 s 4,9 s 1$

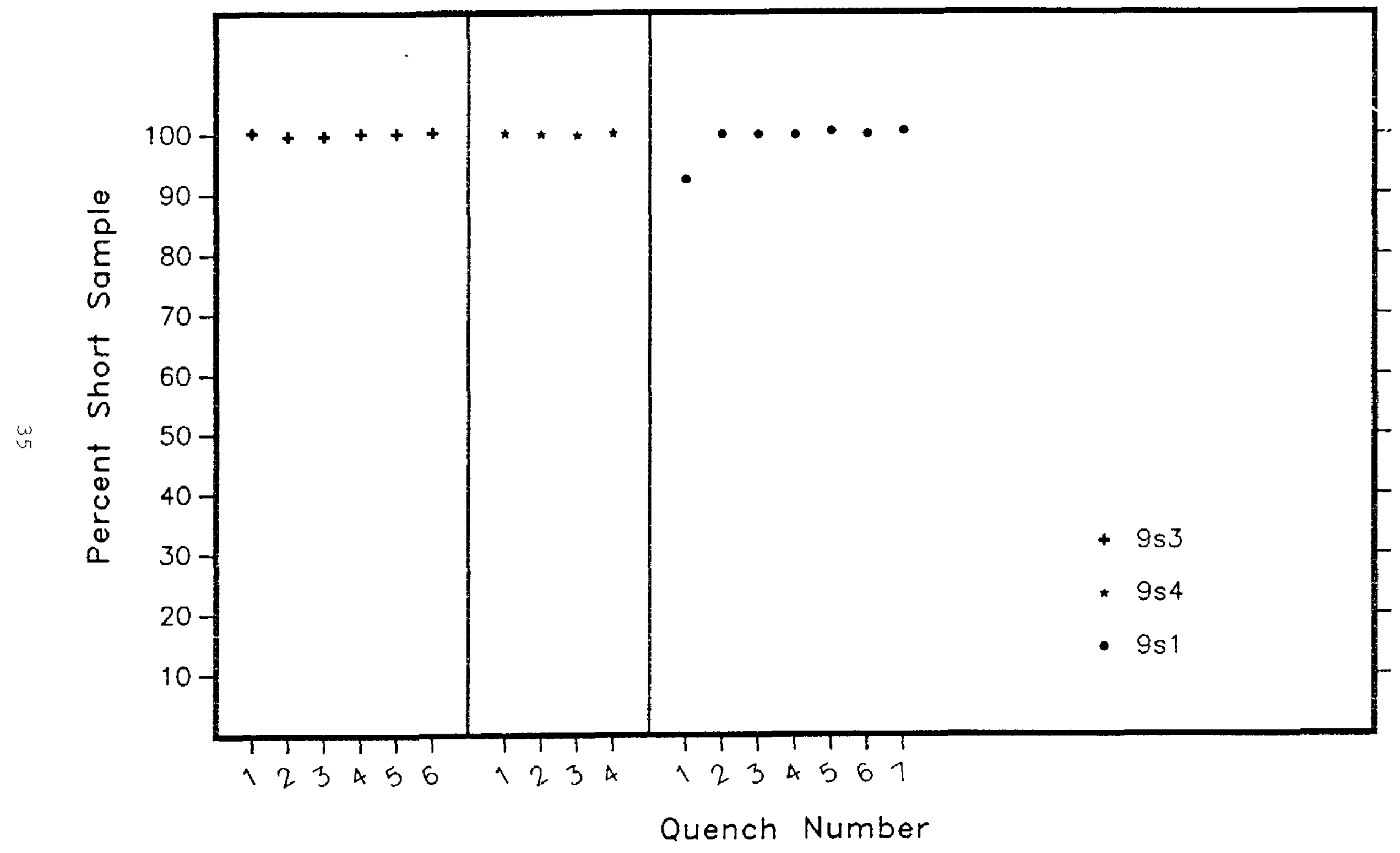

Figure 22

Quench Performance of Three HERA Dipoles 
Operating fIELD

AT HIGHEST FIELD

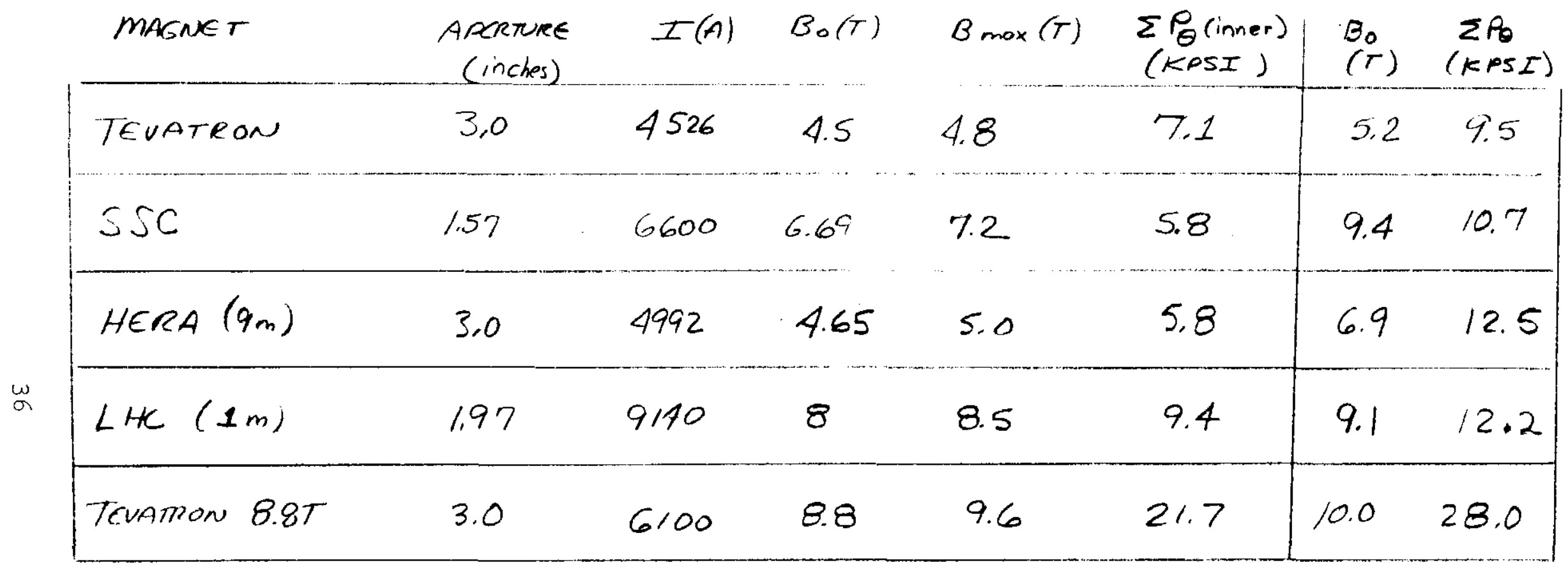

Table III

Comparison of Fields and Inner Coil

Lorentz Pressures for

Recent High-Field Magnets 
Design features of an 8.8 tesla magnet would include

1) The highest current density material available which for high fields appears to be NbTiTa.

2) Kapton insulation with no fiberglass to keep the insulation thickness less than 0.003 inch.

3) Aluminum collars with tapered keys

4) A cold iron yoke designed to support the collared coil assembly

\section{Conclusions}

1) Design considerations for high-field dipoles have been presented.

2) Magnets of $6.6 \mathrm{~T}$ and 3 inch aperture can be built. Magnets with similar field and stress levels have been successfully built.

3) Magnets of $8.8 \mathrm{~T}$ and 3 inch aperture will stretch the current technology well beyond current experience. In particular we are approaching the limits in accommodating magnetic forces in the present geometry. This implies a substantial R\&D program.

4) Proposed program:

a) Initiate the design and fabrication of a conservative $6.6 \mathrm{~T}$ dipole according to the principles outlined.

b) Refine the collared coil assembly above to push performance as close as possible to the $10 \mathrm{~T}$ goal at low temperature.

c) Explore alternate geometries. 


\section{References}

1) Conceptual Design Report, Proton - Proton Collider Upgrade (Main Injector, New Tevatron), Fermi National Accelerator Laboratory, May 1988.

2) Ron Scanlan, presented at the NbTi Workshop, Asilomar, CA, March 1988 (unpublished).

3) W. Sampson, presented at the SSC Magnet System Integration Meeting, Brookhaven National Laboratory, June 17-19, 1988 (unpublished).

4) C. Goodzeit, presented at the SSC Magnet System Integration Meeting, Berkeley, CA, June 2-3, 1987 (unpublished).

5) W. Gilbert and P. Barale, Prompt Report on LBL-SSC Model Magnet D15A S, SSC-MAG-169, November 1, 1987.

6) Gijs de Rijk, CERN (private communication).

7) C. Goodzeit and P. Wanderer, DSS6 Retest with Shims Between Collars and Yoke, SSC-N-510, April 1988.

8) R. Perin, Parameters and Calculated Characteristics of the LHC 8 Tesla Magnet Model (8TM), CERN Internal Note EMA 88/5. 